\title{
Light Source Layout Optimization Strategy Based on Improved Artificial Bee Colony Algorithm
}

\author{
Bo Li, Jue Wang, Zijun Gao, and Ning Gao \\ College of Information Science and Engineering, Dalian Polytechnic University, Dalian, China \\ Correspondence should be addressed to Ning Gao; louisgn@163.com
}

Received 29 June 2021; Revised 3 October 2021; Accepted 11 October 2021; Published 10 November 2021

Academic Editor: Hao Gao

Copyright $\odot 2021$ Bo Li et al. This is an open access article distributed under the Creative Commons Attribution License, which permits unrestricted use, distribution, and reproduction in any medium, provided the original work is properly cited.

\begin{abstract}
In order to optimize the indoor lighting effect and reduce the energy consumption of indoor lighting, the light source layout of conventional rooms and special rooms is studied in this paper. Firstly, the indoor space lighting model is analyzed, and the expressions of light source output and illumination on the target plane are derived. Secondly, in order to improve the search ability of the algorithm in specific areas, combined with the artificial bee colony algorithm, an improved artificial bee colony algorithm suitable for indoor lighting optimization is proposed. Using the average square difference of the illuminance of the target plane as the adaptation function, a penalty function is introduced, and the minimum circle surrounding strategy is used to search the specific location of the light source. Finally, the obtained layout is fitted by engineering standardization, and the indoor light source layout is obtained. Through the simulation of illuminance effect in conventional room and irregular room, it is proved that the proposed method can effectively improve the lighting effect and reduce the energy consumption of indoor lighting under the same number of light sources and the same illuminance index.
\end{abstract}

\section{Introduction}

In today's society, the shortage of energy and the increasing environmental problems are becoming a serious problem that people all over the world need to face. Various energysaving and environmental protection policies have gradually entered people's life. In industrial development, green energy conservation has become the main theme of the development of various industries. Among them, the power resources consumed by the lighting industry account for one-fifth of the whole world every year. This is a terrible resource occupation. If a certain degree of energy can be saved in lighting, it will greatly reduce the pressure of energy conservation and emission reduction in the world. In recent years, the lighting industry is also constantly innovating and developing for green energy conservation. However, most researchers focus on improving the luminous efficiency of the light source and ignore its external energy utilization. A few scholars believe that the lighting and energy-saving methods required for green lighting should not be limited to the traditional luminous efficiency, and the arrangement of light sources may also have a great impact on the lighting and energy-saving effect [1-3]. Therefore, it is necessary to study and strengthen the layout of indoor light sources [4-6].

Aiming at the problems of indoor lighting layout, Komine et al. [7] proposed a typical light source layout, which improved the lighting effect of indoor light source, but this layout caused a lot of energy loss of light source. Yang [8] analyzed the reasonable layout of classroom lighting through the range value and improved the lighting environment but did not conduct in-depth research on the energy loss of the light source. Wei et al. [9] proposed an optimal coverage algorithm based on visible Voronoi diagram to optimize the layout of light sources. The layout can consume less energy to achieve better lighting effect, but it did not demonstrate the uniformity of illumination in detail. Seo et al. [10] improved the design of lighting and daylighting system through the analysis of lighting and daylighting environment, but they lacked the planning of indoor light source layout. Guo and Guo [11] optimized the indoor space layout by improving the small disturbance environment of point light source and realized the system that can adapt to different periods and different weather, but it lacks 
the specific analysis of lighting mode. Wang et al. [12] optimized the layout of traditional indoor lighting by studying the standard deviation of visible light power, but they did not analyze the complex space. Jin and Lee [13] proposed the layout of light source in occupied space based on indoor lighting aesthetics and structure, but it did not actually solve the problem of energy loss of light source. Wang et al. [14] reduced the energy loss of light source by changing the arrangement of near-field LEDs but ignored the influence of illumination uniformity.

In order to solve the problem of energy loss in the light source layout, this paper decides to optimize the indoor light source layout based on the basic idea of artificial bee colony algorithm. After years of development, artificial bee colony algorithm has been a mature optimization algorithm and a specific application of cluster intelligence. It is widely used to solve various problems, such as network traffic prediction and custom prediction [15-17]. In order to solve the above problems, this paper first models and analyzes the indoor lighting and then improves the bee colony optimization algorithm. The feasibility and practicability of the algorithm are verified by verifying the number of light sources and illumination value. It is expected that the algorithm can not only reduce the energy consumption of the light source, but also bring a better lighting experience for users.

The main innovation of this paper is that, by adding penalty function and combining the idea of region segmentation, an improved artificial bee colony algorithm suitable for indoor lighting optimization is proposed, and several conventional rooms and special spaces are simulated and verified $[18,19]$. On the basis of a large number of experiments, it is found that the layout traversed by the algorithm has the data characteristics of certain mathematical functions, so the layout scheme of indoor light source is proposed.

The main contents of this paper are as follows. The second chapter of this paper is to obtain the illuminance relationship of indoor light source on the target plane by modeling and analyzing LED lighting. The work of Chapter 3 is to improve the artificial bee swarm algorithm by combining the illumination relationship of indoor light source with the idea of minimum circle area division. The fourth chapter is about the standardized engineering fitting of the light source layout obtained by the improved algorithm optimization. The content of Chapter 5 is to do a large number of optical data comparison experiments between the fitted light source layout and other classical light source layout. The content of Chapter 6 is to summarize and analyze the new light source layout and the improved artificial bee swarm algorithm, as well as prospects of the future work.

\section{Indoor LED Lighting Model}

The main purpose of this paper is to optimize the layout of indoor light sources, and the premise is to model and analyze the indoor LED lighting model. In the process of modeling, the commonly used energy-saving and environmentfriendly LED light source is selected, and many factors such as brightness, luminous intensity, illumination uniformity, and indoor glare value are comprehensively considered $[20,21]$. Based on the indoor lighting calculation theory [22], the point by point method is used to calculate the receiving plane illumination. The schematic diagram of lighting model is shown in Figure 1.

The room size is set to $L \times W \times H$, the light source is placed at the top of the room, the lower plane is the light source receiving plane, the plane is evenly distributed as $M$ points, and the target plane and the ground height are $H^{\prime}$. The luminous intensity of the light source is

$$
I=I_{0} \cos ^{m} \theta \text {. }
$$

Among them, $I$ is the luminous intensity emitted at angle $\theta, I_{0}$ is the overall luminous intensity of the light source, and $\theta$ is the angle between the line connecting the light source's emission point and the receiving plane and the vector perpendicular to the ceiling; $m$ is the radiation coefficient of the light source, which is related to the light intensity at the half angle, and is controlled by the distance between the midpoint of the curvature of the LED light source part and the package part. Its relationship with the output half angle of the light source is

$$
m=-\frac{\ln 2}{\cos \theta_{1 / 2}} .
$$

At this time, the distance $D_{q}$ between the point $Q\left(x_{q}, y_{q}, H-H^{\prime}\right)$ on the receiving plane and the light source $P(x, y, H)$ is

$$
D_{q}=\sqrt{\left(x_{q}-x\right)^{2}+\left(y_{q}-y\right)^{2}+\left(H-H^{\prime}\right)^{2}} .
$$

Let $H-H^{\prime}=z$, and then, the relationship between $D_{q}$ and $z$ is

$$
D_{q}=\sqrt{\left(x_{q}-x\right)^{2}+\left(y_{q}-y\right)^{2}+z^{2}} .
$$

Among them, $z$ is the vertical distance between the light source and the working surface, and the cosine value of the light source exit angle is

$$
\cos \theta=\frac{z}{D_{q}},
$$

The illuminance $E$ on the receiving plane is

$$
E=\frac{I_{0} \cos ^{m+1} \theta}{D_{q}^{2}}
$$

The illuminance $E_{Q}$ at a certain point on the receiving surface is

$$
E_{Q}=\frac{I_{0} z^{m+1}}{\left[\left(x_{q}-x\right)^{2}+\left(y_{q}-y\right)^{2}+z^{2}\right]^{(m+3 / 2)}} .
$$

Since LEDs are incoherent light sources, when $N$ LEDs are used to form an array, the average light intensity $\bar{E}$ on the receiving plane is 


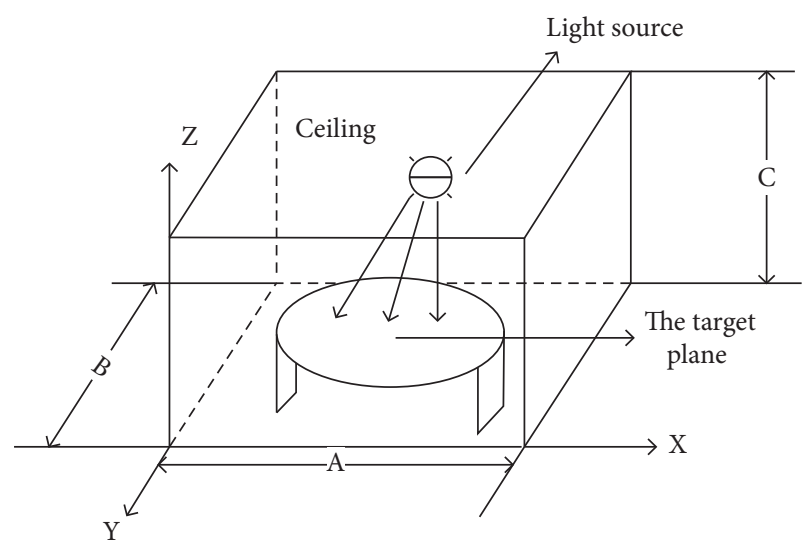

FIgURE 1: Space lighting diagram.

$$
\bar{E}=\frac{1}{M} \sum_{i=1}^{N} \sum_{j=1}^{M} E_{Q}
$$

The mean square error $\sigma$ of the light intensity on the receiving plane is

$$
\sigma=\frac{\sqrt{\sum_{j=1}^{M}\left(E_{Q}-\bar{E}\right)^{2}}}{M} .
$$

This section models the currently commonly used indoor light source LED lighting methods, taking into account the brightness, luminous intensity, uniformity of illuminance, and indoor glare values that affect indoor lighting and many other factors. Through the analysis and calculation of the LED light intensity and the spatial distance, the illuminance relation expression of the light source on the target plane is derived.

\section{Algorithm Optimization Module of Light Source}

According to the indoor lighting standard, the indoor illumination value should be in the range 300-1500 lx, and the illumination uniformity should be greater than 0.7 . In order to realize the concept of green energy-saving lighting, the use of light sources should be reduced under the premise of ensuring the indoor lighting standard. Therefore, this paper attempts to optimize the location of the light source layout, on the premise of meeting the illumination and illumination uniformity, optimize the light source layout, and use the least light source to achieve the optimal lighting effect.

At present, the commonly used algorithms to solve optimization problems include genetic algorithm, ant colony algorithm, particle swarm optimization, artificial bee colony, and other intelligent optimization algorithms, as well as mathematical optimization algorithms. Artificial bee colony algorithm has the advantages of high accuracy, strong robustness, and strong exploration ability and has no high requirements for the objective and constraint function [23-29]. Based on the optimization idea of artificial bee colony algorithm and the algorithm structure of region segmentation, an improved artificial bee colony algorithm for indoor light source layout optimization is proposed.
The improved artificial bee colony algorithm treats the layout of the light source as a solution in a multidimensional space. The position of each light source has a unique corresponding nectar source, and the position of each nectar source represents a feasible solution to the problem. The number of guide bees in the population is the same as the number of follower bees, each accounting for one-half of the number of nectar sources, and each nectar source corresponds to only one guide bee at the same time. In the initialization stage, the nectar source is generated by random numbers in the optimization interval, the LED lighting function is established by equation (7), the evaluation function is constructed by equation (9), and the nectar source in the population is initialized according to the number of light sources. The value in each dimension represents relative coordinates of the light source. In the optimization stage, the area is divided into uniform grids and assigned corresponding binary numbers to determine the light source code represented by the position. In the search stage, after the bee finds a new source of nectar, it shares the information source with the bee, and the bee uses the shared information to follow the bee probabilistically using roulette. In the iterative process, the mean square error of the lighting function is set as the fitness value. The larger the grid area occupied by the nectar in the space, the greater the fitness, and vice versa. The nectar source is updated and replaced by adopting a greedy selection strategy. If the new nectar source does not meet the standard or has reached the search threshold and no suitable nectar source is found, the nectar source location is abandoned, and a new nectar source is randomly generated and iteratively searched using the objective function and the evaluation function. When the fitness reaches the set satisfaction, the output current honey source parameters are the current optimized relative coordinates of the light source.

Under the requirement of fast convergence, the processing level of artificial bee colony algorithm in special space decreases. When the required optimization space is an irregular room, at the later stage of the iteration of the conventional artificial bee colony algorithm, most optimization particles will repeatedly appear near the same area, resulting in some overlapping areas of light sources, resulting in the loss of light source energy.

Firstly, in order to facilitate the processing of engineering problems, the artificial bee colony algorithm is improved, and the penalty function and dynamic penalty factor are added to the algorithm. The equations are shown as follows:

$$
\begin{array}{r}
\min f(s), \\
\begin{cases}\text { s.t. } g_{i}(s) \geq 0, & i=1, \ldots, u, \\
h_{j}(s)=0 . & j=1, \ldots, v,\end{cases} \\
\min F(s, \lambda)=f(s)+\lambda P(s), \\
P(s)=\sum_{i=1}^{u} \mu\left(g_{i}(s)\right)+\sum_{j=1}^{v} \sigma\left(h_{j}(s)\right) .
\end{array}
$$

Among them, $F(s, \lambda)$ is a penalty function. $f(s)$ is an objective function. $\lambda P(s)$ is a penalty term. $\lambda$ is a dynamic penalty factor. $g_{i}(s)$ and $h_{j}(s)$ are constraint functions. $\mu$ and 


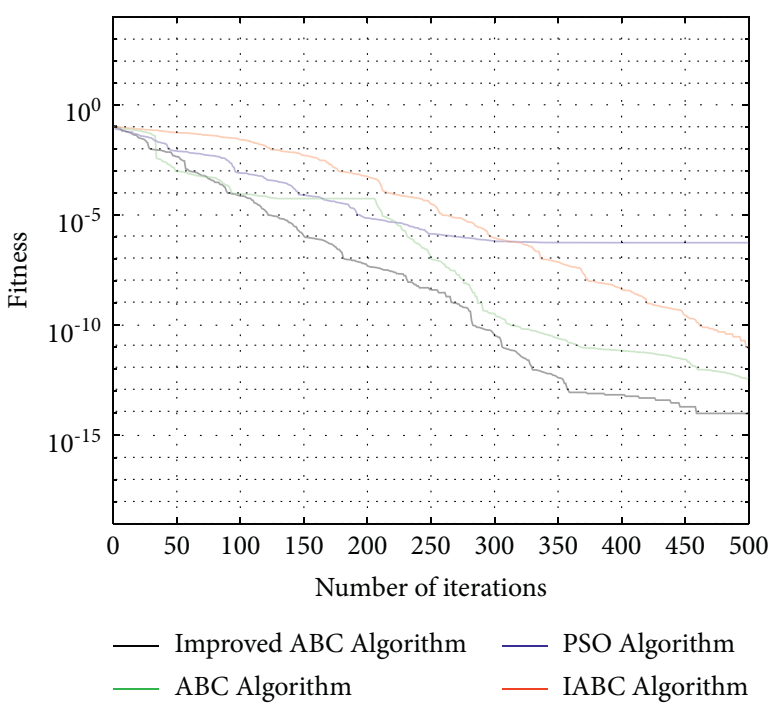

(a)

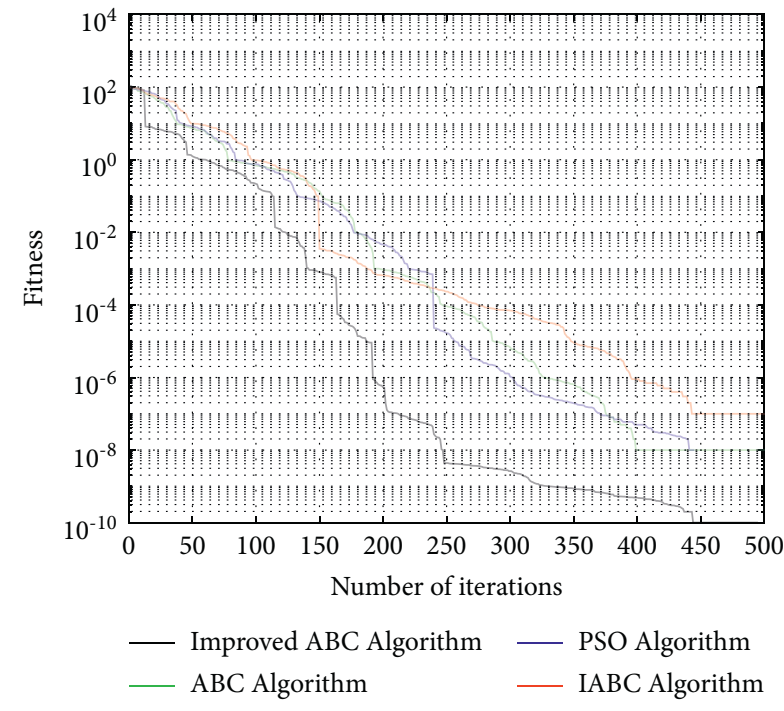

(b)

Figure 2: Fitness trend chart under test functions Griewank and Rastrigin. (a) Griewank function. (b) Rastrigin function.

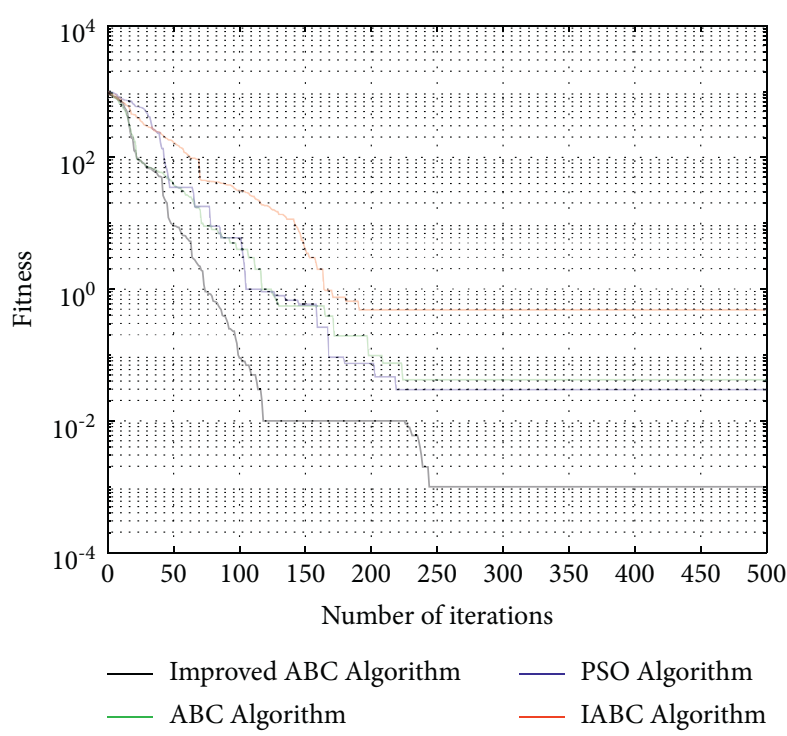

(a)

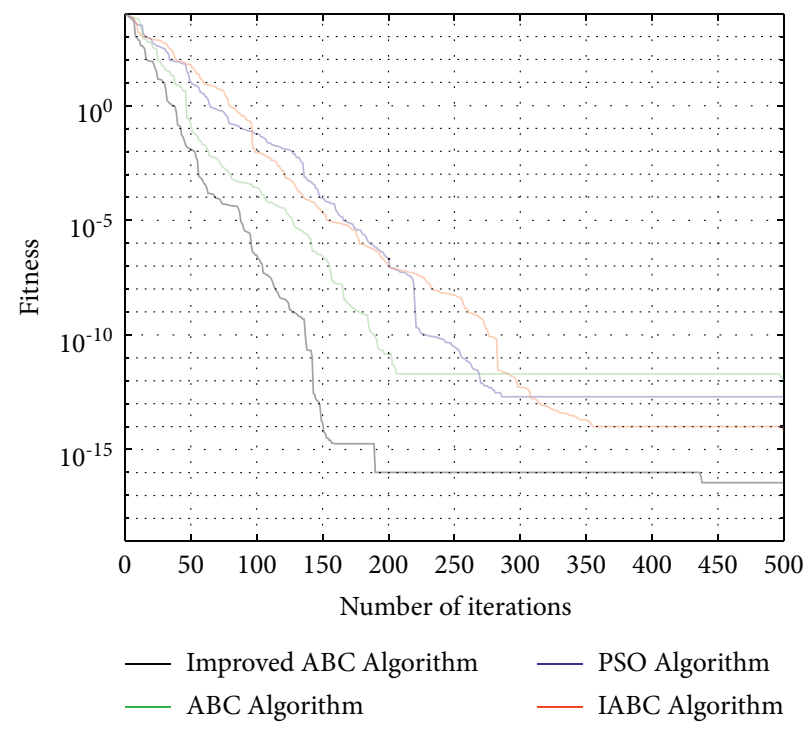

(b)

FIGURE 3: Fitness trend chart under test functions Rosenbrock and sphere. (a) Rosenbrock function. (b) Sphere function.

$\sigma$ are functions of $g_{i}(s)$ and $h_{j}(s)$. In order to facilitate the implementation of the algorithm, let the constraint equation and inequality take the same dynamic penalty factor, and the value of the dynamic penalty factor is shown in

$$
\lambda=10^{\left.e^{\left(c_{2}-c_{1} / 5-20\right.}\left(G_{2} / G_{1}\right)\right)+C_{1}+1}
$$

where $c_{1}$ and $c_{2}$ are fixed constants; $c_{1}=4, c_{2}=6$ are selected in this paper; $G_{1}$ and $G_{2}$ are the maximum iteration value and the current iteration value, respectively.

Firstly, by adding a penalty function in the algorithm, the optimization problem of light source position is changed into an unconstrained problem, and the unconstrained optimal solution of light source position is equivalent to the optimal solution of the initial problem to reduce the difficulty of light source position optimization. At the same time, the dynamic penalty factor is used to control the global convergence of the algorithm. In the initial stage of population iteration, the penalty factor is very small, which makes the individual distribution and search dimension in the population wide, which is conducive to the realization of global search. In the middle and late stage of population iteration, with the increase of feasible solution set, the penalty factor increases exponentially, which makes the algorithm tend to converge to the optimal solution range. 


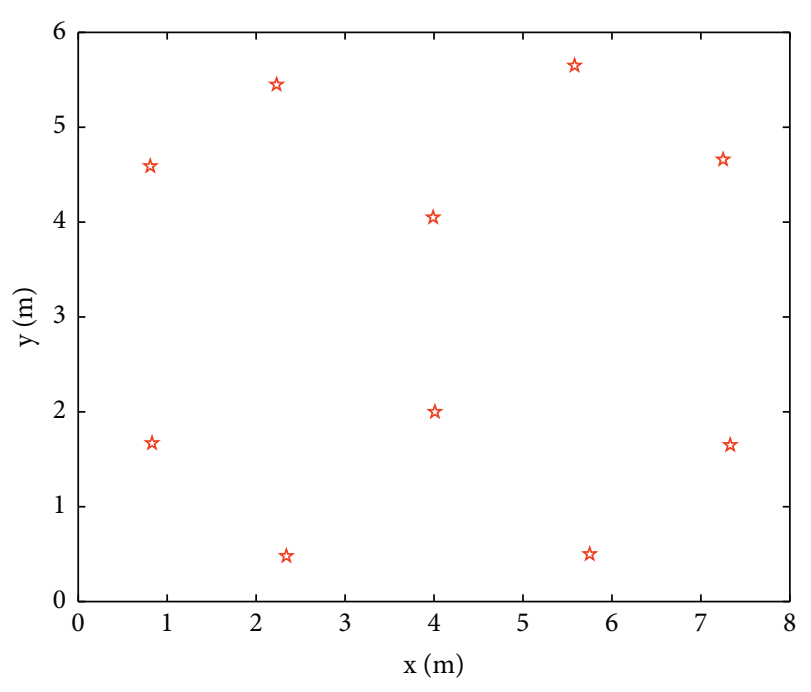

th Light source under improved $\mathrm{ABC}$ algorithm

Figure 4: $8 \times 6 \times 3 \mathrm{~m}$ room light source layout.

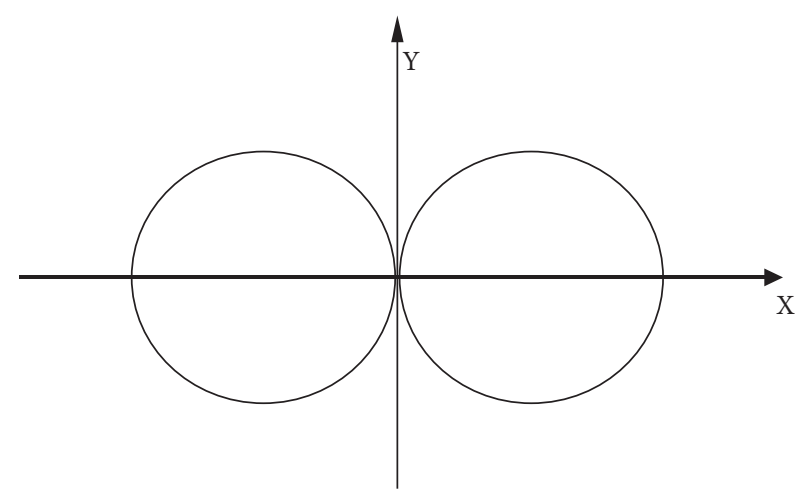

Figure 5: Bernoulli line.

Secondly, aiming at the problem of energy loss of light source in irregular space, region segmentation is added to artificial bee colony algorithm to separate the superimposed regions. In order to reduce the interaction between the light superposition area and the conventional area, the algorithm is set to calculate the illumination distribution of the conventional area first. According to the light superposition area, the minimum circle area coverage method is used to search the minimum light source coverage area.

In view of the characteristics that the light source weakens continuously with distance and involves the lighting of light sources in irregular areas, because the idea of region segmentation and minimum circle optimization algorithm in this paper is to use points to divide the irregular space $Z Q$ for the layout of light sources, set $Z P$ as the best solution set in region segmentation, where

$$
Z Q(a)=\{b \in Z \mid(\overline{a b} \subset Z)\} .
$$

If there is no intersection between points $a$ and $b$ in the irregular area $Z$ in the space and outside the $Z$ area, $a$ and $b$ are regarded as exactly the same visible points in the same

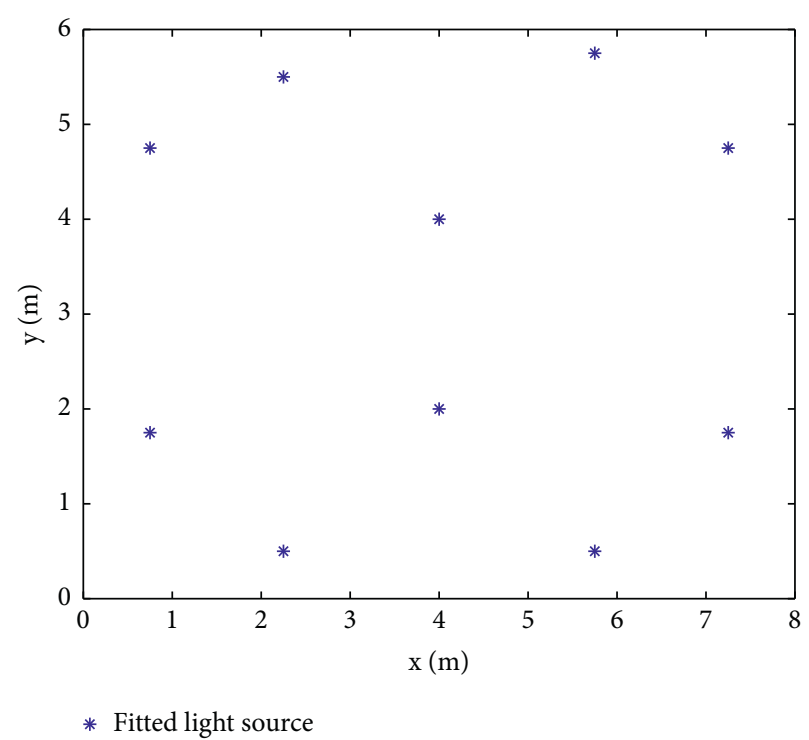

Figure 6: $8 \times 6 \times 3 \mathrm{~m}$ room fitting layout.

space. Therefore, the same region of a point $a$ in the irregular region $Z$ is defined as the set $(Z Q)$ of complete intersections of all points starting from $a$ and intersecting at the same point in the irregular region $Z$.

$$
Z P(A)=\bigcup_{i=1}^{n} Z R\left(a_{i}\right)
$$

where $A$ is a set of $n$ points, the positions between the $n$ points are completely independent, and $Z R$ is the completely irregular region $(Z P)$ of point of $A$. That is,

$$
Z R\left(a_{i}\right)=\left\{b \in Z \mid\left\|b-a_{i}\right\| \leq\left\|b-a_{j}\right\|, \forall j \neq i, a_{i}, a_{j} \in A\right\},
$$

$\left\|b-a_{i}\right\|$ represents the Euclidean distance between the $b$ point and the point $a$ in the irregular area.

$$
Z D(A)=\bigcup_{i=1}^{n} Z X\left(a_{i}\right)
$$

$Z D$ is the same visible figure of the whole set of $A$, where

$$
Z X\left(a_{i}\right)=\left\{b \in Z Q\left(a_{i}\right)\left\|b-a_{i}\right\| \leq\left\|b-a_{j}\right\|, \forall j \neq i, b \in Z Q\left(a_{i}\right)\right\},
$$

is point $a_{i}$ exactly the same as that of the visible area $(Z X)$.

Therefore, when calculating the visible area illuminated by the light source, based on the environmental conditions constructed in Chapter 2, the relationship between the distances of points $a$ and $b$ in the irregular area $Z$ is as follows:

$$
f(a, b)= \begin{cases}\|b, a\|, & b \in Z Q(a), \\ \infty, & b \notin Z Q(a) .\end{cases}
$$

The minimum distance between adjacent iterative particles is constrained by the distance formula to ensure the accuracy of the final result. 
After combining the penalty function and region segmentation:, the execution steps of the improved artificial bee colony algorithm in the search space are as follows:

(i) Step 1. Judge whether the target space is a regular area. If it is a regular area, go to step 3 . When the target space is a complex area, go to step 2.

(ii) Step 2. Divide the complex area, detect the overlap area of the light source, calculate the minimum coverage area $f$ the overlap area, and step into Step 3.

(iii) Step 3. Discrete precalculated area light source initial position iteration number.

(iv) Step 4. Establish the objective function and fitness function according to equations (7) and (9).

(v) Step 5. Calculate the respective density of the binary numbers of the light source code, and establish the relative coordinates of the light source in the space corresponding to the value in each dimension of the nectar source.

(vi) Step 6. Iteratively solve the fitness value of the light source position represented by the nectar, adopt the strategy of greedy selection, compare with the fitness value of the best light source position of the previous generation, decide to eliminate or select the better solution, and calculate the follow probability at the same time.

(vii) Step 7. Update the optimal position and following probability of the light source in the population.

(viii) Step 8. Iteratively replace the dimension value of the light source position.

(ix) Step 9. If the expected conditions are met or the maximum number of iterations is reached, stop the search and judge whether the standard is met. If the expected condition is not reached, and the search threshold is reached, step 3 is executed; if the condition is met, the light source position is output.

In order to visually compare the performance of the algorithm, four different functions, Sphere, Rastrigin, Rosenbrock, and Griewank, are used to test the improved artificial bee swarm algorithm, the original artificial bee swarm algorithm, the particle swarm algorithm, and the IABC algorithm improved in document [30].

The simulation experiment uses MATLAB 2019a as the experimental platform, the operating system is Windows 10 , the memory is $16 \mathrm{G}$, the CPU is Intel(R) Core(TM) i5-9400, and the main frequency is $2.9 \mathrm{GHz}$. To avoid the randomness of the optimization algorithm, this experiment will use the average value of the results obtained by running the program 30 times as the final fitness curve result. The expression and value range of each test function are shown in Table 1 . The fitness change trend of the standard test function of each algorithm is shown in Figures 2 and 3.

In Figures 2 and 3, the improved artificial bee swarm algorithm uses a new fitness function and a region search scheme compared with the original artificial bee swarm algorithm, particle swarm algorithm, and IABC algorithm mentioned in document [30], which can not only search for the optimal location more quickly, but also avoid the local optimum caused by multiple particle overlap at the end of iteration.

\section{Optimization Results Engineering Standardized Position Fitting}

In this paper, $8 \times 6 \times 3 \mathrm{~m}$ general specification room is calculated. After optimization by improved artificial bee colony algorithm, the relative coordinates of light source are shown in Table 2, and its spatial layout is shown in Figure 4.

In Figure 4, the left half of the space is mainly illuminated by No. 3, No. 4 , No. 5 , and No. 6 light sources, the right half of the space is mainly illuminated by No. 7, No. 8, No. 9, and No. 10 light sources, and the central area is mainly illuminated by No. 1 and No. 2 main light sources and edge light sources. Among them, the $x$-axis coordinates of No. 1 and No. 2 light sources, No. 3 and No. 6 light sources, No. 4 and No. 5 light sources, No. 7 and No. 10 light sources, and No. 8 and No. 9 light sources are inconsistent in the spatial coordinate system, while the $y$-axis coordinates of No. 6 and No. 7 light sources, No. 5 and No. 8 light sources, No. 4 and No. 9 light sources, and No. 3 and No. 10 light sources are inconsistent in the spatial coordinate system, and there are some differences.

The analysis of the optimized light source layout position shows that although the lighting effect achieved by this layout can meet the actual lighting requirements, it has relatively random characteristics, and the coordinates of the light source layout are asymmetrical, which lacks aesthetics in the actual layout. In this paper, the regular curve equation is used to further study the optimization results, so as to achieve the beauty, symmetry, and engineering practicability of the layout. Through a large number of experimental analysis, it is found that the shape formed by the actual coordinate position of the light source is similar to the shape of Bernoulli Line in mathematics. Therefore, this paper uses the Bernoulli Line to fit the indoor light source layout in engineering standardization.

The Bernoulli Line is a curve in the plane rectangular coordinate system. It is the inversion figure of hyperbola about the circle whose center is in the hyperbolic center. Its mathematical expression is shown in equation (18).

$$
\left(x^{2}+y^{2}\right)^{2}=2 a^{2}\left(x^{2}-y^{2}\right) \text {. }
$$

The mathematical graph is shown in Figure 5.

In the case of a limited number of interpolations, this paper uses the principle of least squares to perform fitting operations [31-33]. The least square method matches the best function of the data by minimizing the sum of squares of errors. The fitting function is as follows:

$$
\alpha x^{4}+\beta y^{4}+\delta x^{2} y^{2}+\gamma x^{2}-\eta y^{2}+\varphi .
$$

In combination with the least square method, equation (20) is as follows: 
TABLE 1: Expression and range of test function.

\begin{tabular}{lcc}
\hline Function name & Function expression & Search scope \\
\hline Griewank & $f_{1}(x)=\sum_{i=1}^{n} x_{i}^{2}$ & {$[-600,600]$} \\
Rastrigin & $f_{2}(x)=\sum_{i=1}^{n}\left(x_{i}^{2}-10\left(\cos \left(2 \pi x_{i}\right)\right)+10\right)$ & {$[-5.12,5.12]$} \\
Rosenbrock & $f_{3}(x)=\sum_{i=1}^{n} 100\left(x_{i+1}-x_{i}^{2}\right)^{2}+\left(1-x_{i}\right)^{2}$ & {$[-100,100]$} \\
Sphere & $f_{4}(x)=(1 / 4000) \sum_{i=0}^{n} x_{i}^{2}-\prod_{i=1}^{n} \cos \left(x_{i} / \sqrt{i}\right)+1$ & {$[-100,100]$} \\
\hline
\end{tabular}

TABLE 2: Relative coordinates of $8 \times 6 \times 3 \mathrm{~m}$ room light source.

\begin{tabular}{|c|c|c|c|c|c|}
\hline Light source number & Coordinate $X(m)$ & Coordinate $Y(m)$ & Light source number & Coordinate $X(m)$ & Coordinate $Y(m)$ \\
\hline 1 & 3.99 & 4.05 & 6 & 2.23 & 5.45 \\
\hline 2 & 4.01 & 2.00 & 7 & 5.58 & 5.65 \\
\hline 3 & 2.34 & 0.48 & 8 & 7.25 & 4.66 \\
\hline 4 & 0.83 & 1.67 & 9 & 7.33 & 1.65 \\
\hline 5 & 0.81 & 4.59 & 10 & 5.75 & 0.50 \\
\hline
\end{tabular}

$$
\left\{\begin{array}{l}
R_{1}=[\alpha, \beta, \delta, \gamma, \eta, \varphi]^{T}, \\
R_{2}=\left[x^{4}, y^{4}, x^{2} y^{2}, x^{2}, y, 1\right]^{T}
\end{array}\right.
$$

The optimization goal is as follows:

$$
\begin{cases}\min & \left\|R_{1}^{T} R_{2}\right\|^{2}=R_{1}^{T} R_{2} R_{2}^{T} R_{1}, \\ \text { s.t. } & R_{1}^{T} U R_{1}<0,\end{cases}
$$

where $R_{1}^{T} U R_{1}<0$ is constrained by the properties of the double helix: $a c-b^{2}<0$.

The layout of engineering standardized fitting light source after smoothing is shown in Figure 6. Comparing Figure 6 with Figure 4, it can be seen that the layout position of the light source has not changed greatly, but its symmetry and aesthetics are greatly enhanced.

In this section, on the premise of aesthetics and engineering practicability, through the analysis of the optimal light source layout position optimized by improved artificial bee colony algorithm, and using the mathematical curve equation to carry out the standardized fitting of the light source position, the engineering standardized fitting light source layout is obtained.

\section{Simulation Experiment}

In order to verify the rationality and superiority of the indoor space lighting layout proposed in this paper, four aspects of simulation experiments are carried out. Simulation experiment 1 is used to verify the difference of lighting effect between the engineering standard fitting light source layout and the optimal light source layout optimized by improved artificial bee colony algorithm. Simulation experiment 2 is used to verify the difference between the indoor light source layout finally given by this method and the indoor light source layout lighting effect given by other documents. Simulation experiment 3 is used to verify the energy consumption comparison between the final light source layout given by this method and the indoor light source layout given by other documents at the same illuminance effect. Simulation experiment 4 is used to verify the application effect of this method in irregular rooms. In simulation experiments 1,2 , and 3 , in order to reflect the generality of the method, standard square room, medium rectangular room, and large rectangular room are selected. Three room sizes of $5 \mathrm{~m} \times 5 \mathrm{~m} \times 3 \mathrm{~m}, 8 \mathrm{~m} \times 6 \mathrm{~m} \times 3 \mathrm{~m}$, and $12 \mathrm{~m} \times 9 \mathrm{~m} \times 3 \mathrm{~m}$ are selected as examples. In Simulation Experiment 4, an irregular space of L-shaped room is selected as an example.

5.1. Simulation Experiment 1. In order to verify the difference of lighting effect between the engineering standard fitting light source layout and the optimal light source layout optimized by improved artificial bee colony algorithm, this experiment compares and analyzes the fitting accuracy and illumination distribution. In terms of fitting accuracy, the least square fitting method of boundary [34] is used to calculate the fitting layout accuracy of rooms with various specifications. In terms of illuminance distribution, the same number of light sources is used in the same room to compare the illuminance distribution of multiple groups of different room specifications. The layout comparison diagram and illuminance distribution diagram are shown in Figures 7-9.

In Figures 7-9, the layout of engineering standardized fitting light source deviates slightly from the layout directly optimized by using the improved artificial bee colony algorithm. The boundary difference is obtained by traversing the sampling coordinates of the fitting layout and the results optimized by the algorithm. It can be proved that the error rate of the two layouts is very small, which meets the requirements of boundary fitting threshold. Through the comparative analysis of indoor illuminance, under the same room standard and the same number of light sources, although the lighting effect of the optimized light source layout using the improved artificial bee colony algorithm is slightly better, the illuminance deviation from the engineering standardized fitting light source layout is very small, which can be ignored. The lighting effects of the two are consistent, and the light source layout of engineering standardized fitting is more symmetrical and beautiful, 


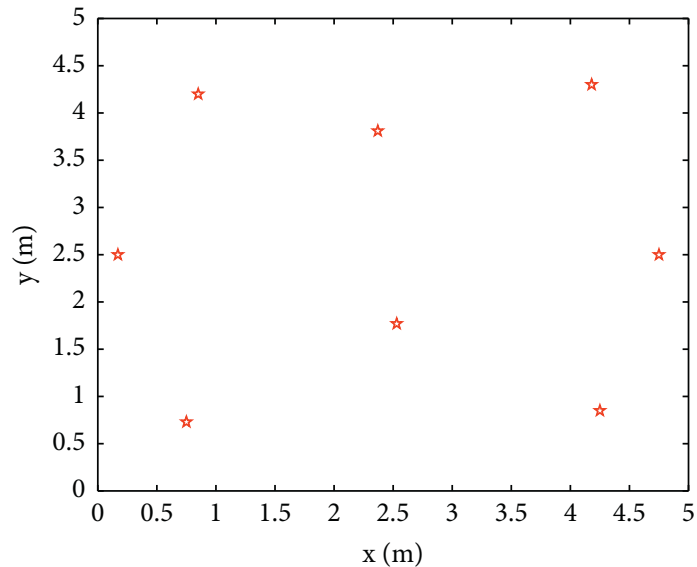

* Light source under improved $\mathrm{ABC}$ algorithm

(a)

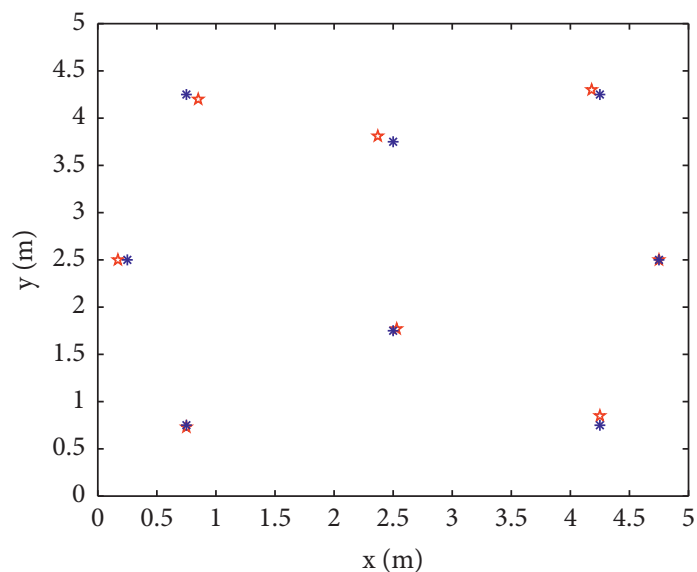

\ Light source under improved $\mathrm{ABC}$ algorithm

* Fitted light source

(c)

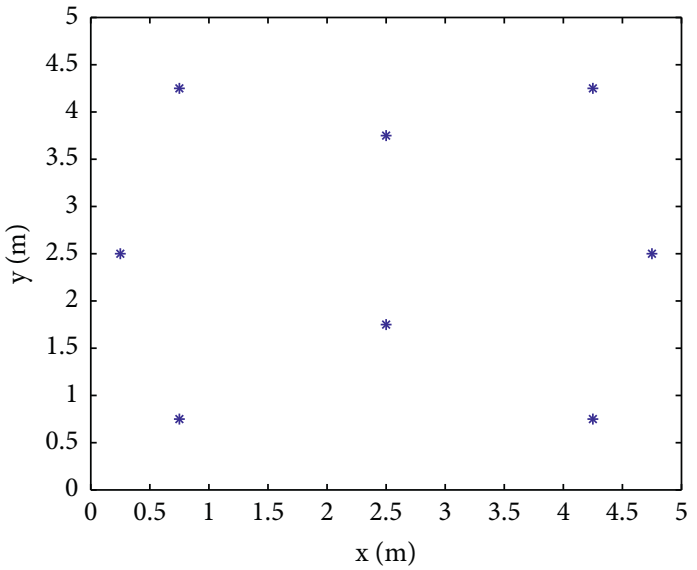

* Fitted light source

(b)

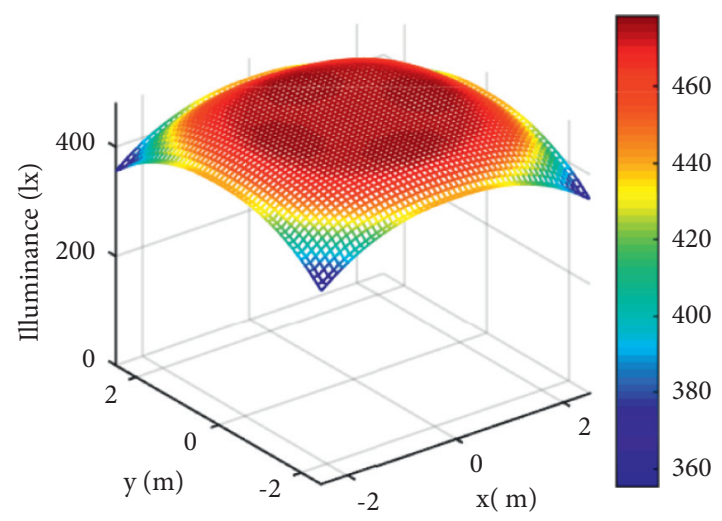

(d)

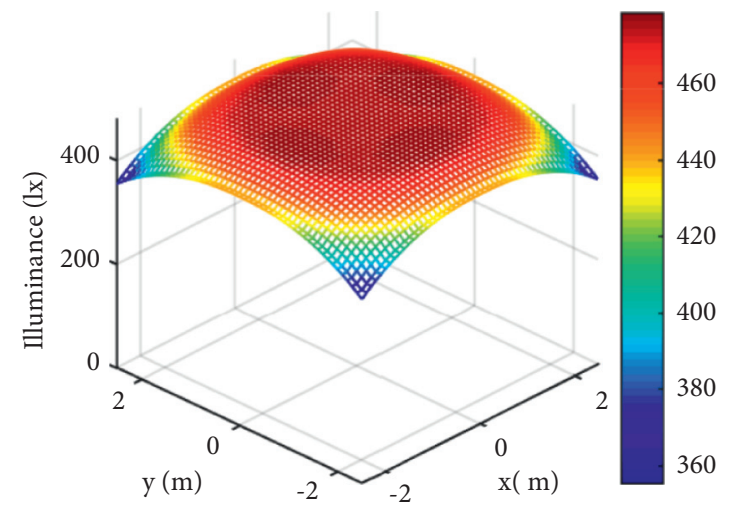

(e)

Figure 7: $5 \mathrm{~m} \times 5 \mathrm{~m} \times 3 \mathrm{~m}$ room layout and illumination diagram. (a) Optimized layout. (b) Fitted layout. (c) Layout comparison. (d) Fitting layout illumination map. (e) Optimized layout illumination map.

which is convenient for construction. It verifies the practicability of the improved artificial bee colony algorithm in this paper and the rationality of its optimization results after engineering standardized fitting.
5.2. Simulation Experiment 2. This simulation experiment is used to verify the difference between the indoor light source layout finally given by this method and the lighting effect of the indoor light source layout given by other 


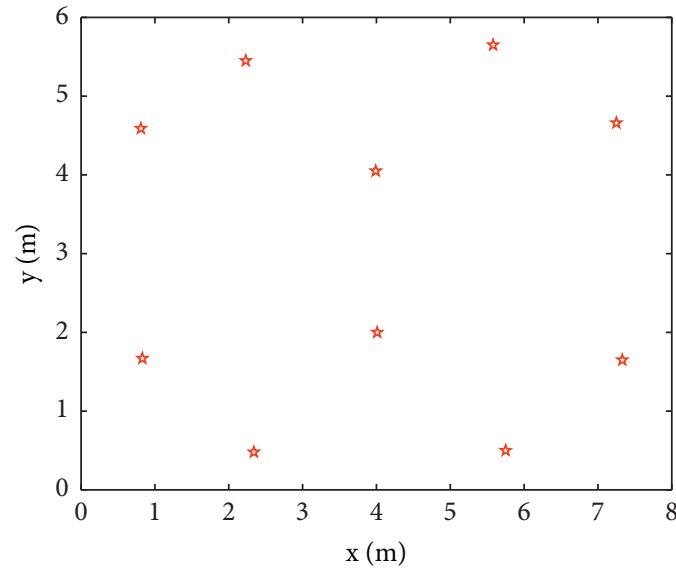

th Light source under improved $\mathrm{ABC}$ algorithm

(a)

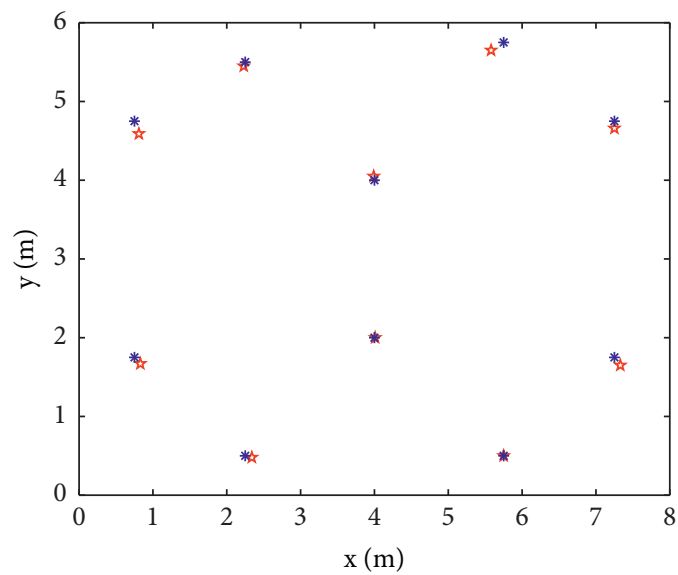

* Light source under improved ABC algorithm

* Fitted light source

(c)

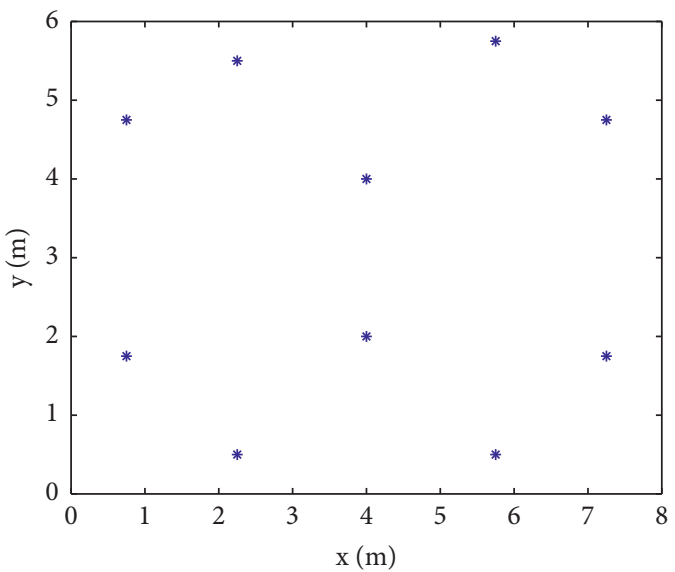

* Fitted light source

(b)

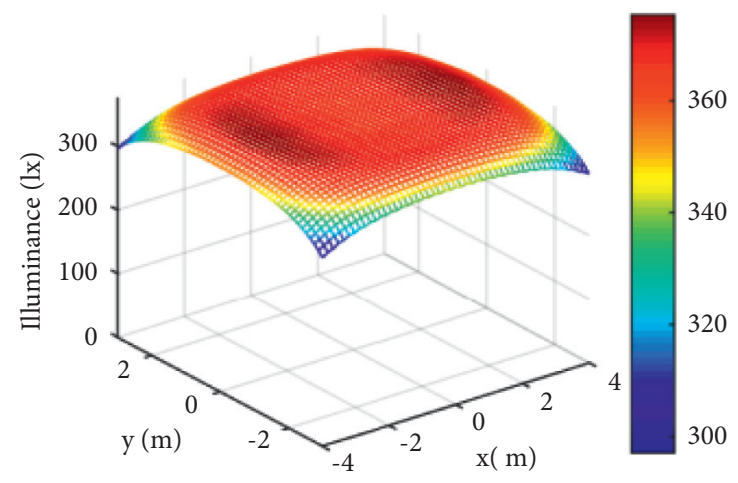

(d)

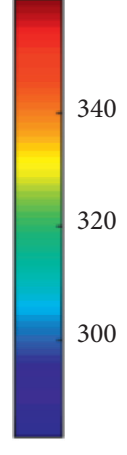

340

20

300

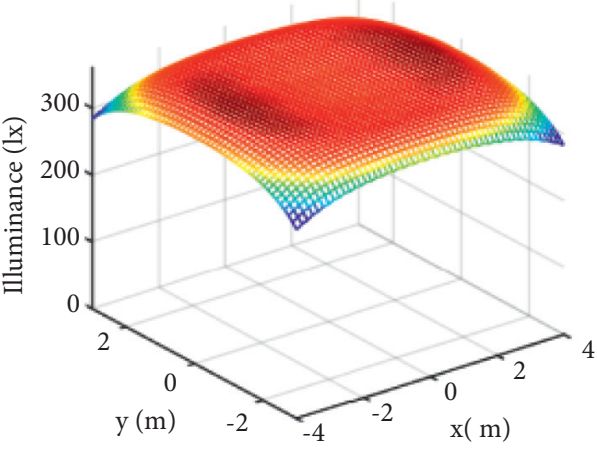

(e)

FigURE 8: $8 \mathrm{~m} \times 6 \mathrm{~m} \times 3 \mathrm{~m}$ room layout and illumination diagram. (a) Optimized layout. (b) Fitted layout. (c) Layout comparison. (d) Fitting layout illumination map. (e) Optimized layout illumination map.

documents. According to the light source layout and parameters given in reference [35], the lighting effects of rectangular and circular light source layouts are simulated by using the same number of light sources in the same room. The layout of the light source and its illuminance are shown in Figure 10; $8 \mathrm{~m} \times 6 \mathrm{~m} \times 3 \mathrm{~m}$ is shown in Figure 11; and $12 \mathrm{~m} \times 9 \mathrm{~m} \times 3 \mathrm{~m}$ is shown in Figure 12. The indoor light source layout and lighting effects finally given by the method in this paper are shown in Figures 7-9 in Section 5.1 , respectively.

Take a $5 \mathrm{~m} \times 5 \mathrm{~m} \times 3 \mathrm{~m}$ room as an example to analyze the experimental results. In Figure 10, the maximum 


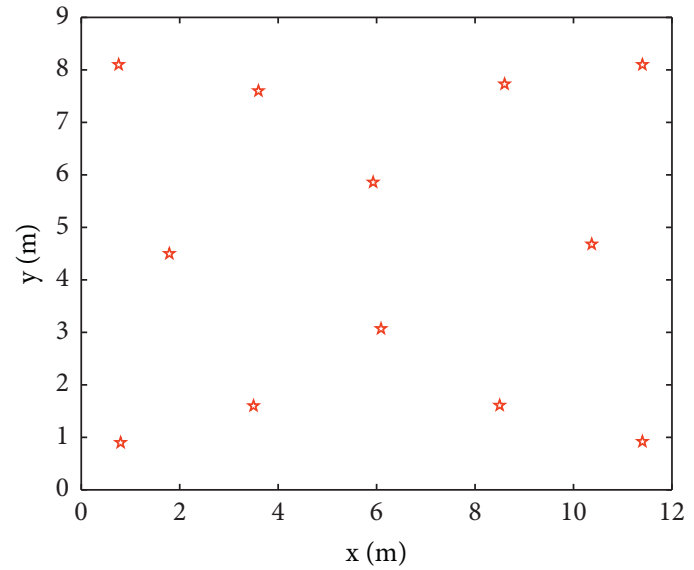

\ Light source under improved $\mathrm{ABC}$ algorithm

(a)

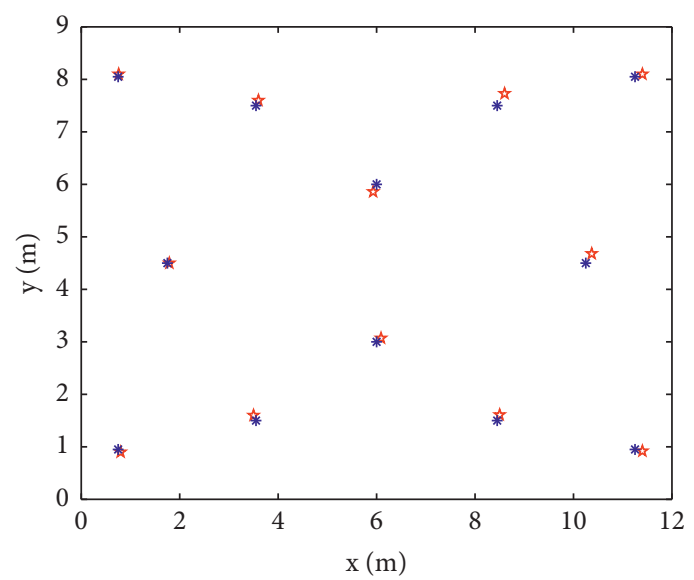

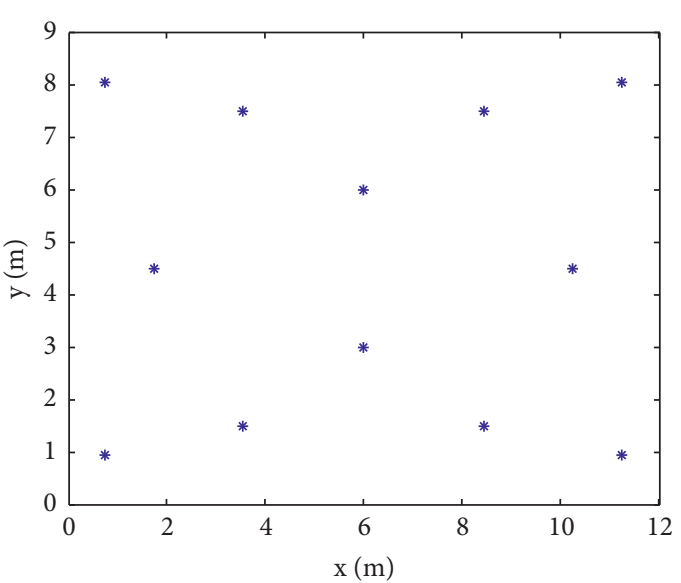

* Fitted light source

(b)

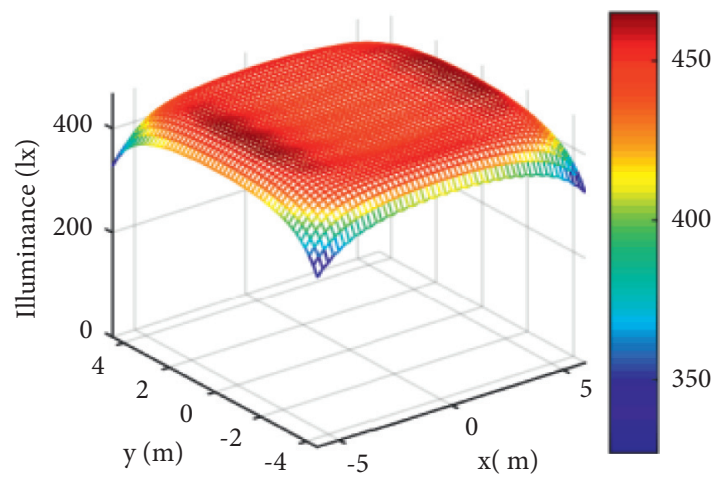

(d)

(c)

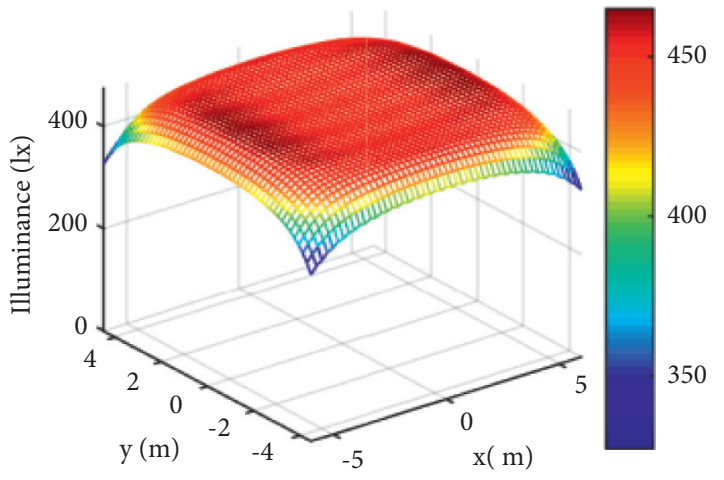

(e)

FIGURE 9: $12 \mathrm{~m} \times 9 \mathrm{~m} \times 3 \mathrm{~m}$ room layout and illumination diagram. (a) Optimized layout. (b) Fitted layout. (c) Layout comparison. (d) Fitting layout illumination map. (e) Optimized layout illumination map.

indoor illuminance value of the rectangular layout is $555.164 \mathrm{~lx}$, the minimum is $288.548 \mathrm{~lx}$, the average room illuminance is $388.879 \mathrm{~lx}$, and the uniformity of illuminance is $74.2 \%$. Although the rectangular layout can meet the indoor lighting requirements in terms of illuminance value, there is a great difference between the maximum and minimum values of illuminance. In terms of lighting effect, there is a big gap between the central part and the edge part 


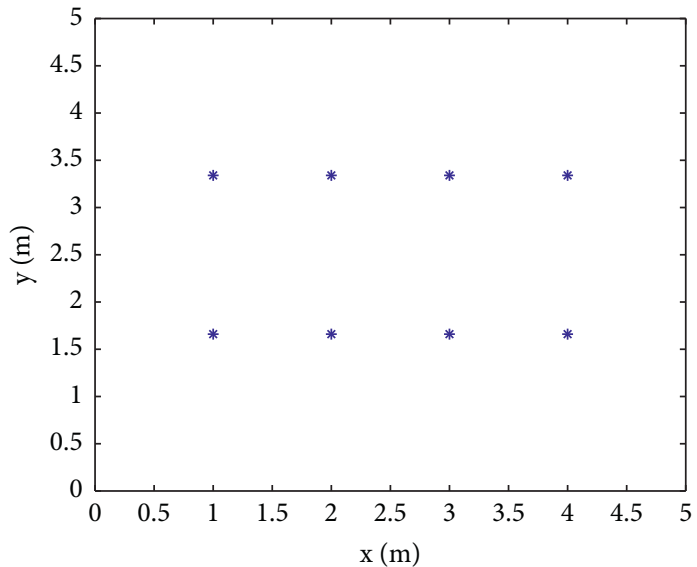

* Light source

(a)

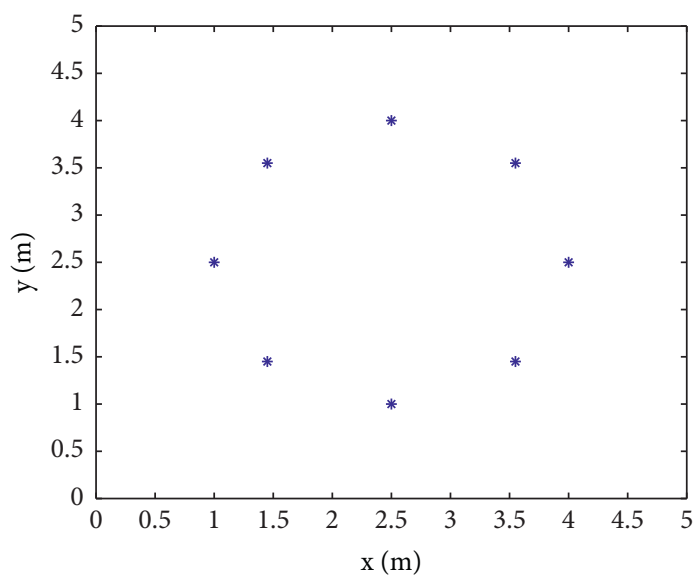

* Light source

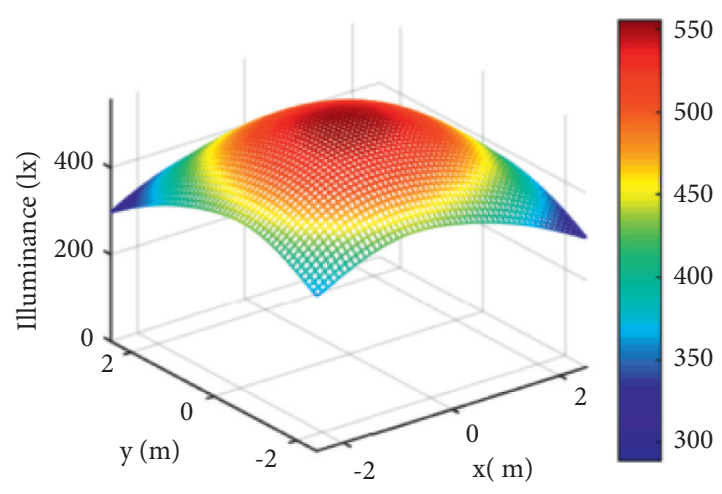

(b)

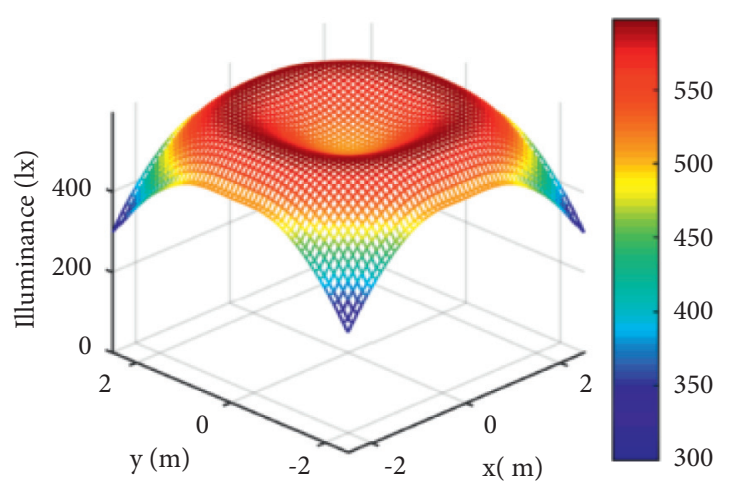

(d)

FIGURE 10: $5 \mathrm{~m} \times 5 \mathrm{~m} \times 3 \mathrm{~m}$ room layout and illumination diagram. (a) Layout of rectangular light source. (b) Rectangular layout illumination map. (c) Layout of circular light source. (d) Circular layout illumination map.

of the rectangular light source layout. The illumination value changes obviously from the center to the edge, and the room illumination distribution is unbalanced, which leads to the characteristics of high central illumination and low edge illumination, which affects the overall lighting effect.

In Figure 10(d), the light source is arranged in a traditional circular layout, the maximum illumination is $596.7869 \mathrm{~lx}$, the minimum is $299.3843 \mathrm{~lx}$, the average illumination in the room is $395.488 \mathrm{~lx}$, the difference between the maximum and minimum illumination is $297.4026 \mathrm{~lx}$, and the illumination uniformity is $75.7 \%$. In the layout of circular light source, the central illumination value is too high, and the edge illumination value is too low, which shows a cliff like downward trend at the corner of the space, so that the uniformity of room illumination is at a low level, resulting in the uneven distribution of indoor illumination. Circular layout exposes the problem of sharp decrease of illumination value at the edge of space, which has a great impact on personnel activities.
In Figure 7, the maximum illuminance value of the space simulated by the method in this paper is $438.8067 \mathrm{~lx}$, the minimum illuminance value is $355.2899 \mathrm{~lx}$, the average indoor illuminance is $410.953 \mathrm{~lx}$, and the difference between the maximum and minimum illuminance is $83.5168 \mathrm{~lx}$. The uniformity is $86.4 \%$, which is higher than the lighting standard. In terms of illuminance value, the fitting layout is smaller in illuminance difference, the illuminance change from the central area to the edge area is more gentle, the overall illuminance value decreases less, and there is no sharp decrease in the edge illuminance value, which reduces the local high illuminance uniformity and makes the lighting effect more uniform.

In rooms of different specifications, the illuminance distribution changes with the change of the light source layout. The uniformity of the illuminance of each layout under the same number of light sources is shown in Table 3 .

In Table 3, under the same room standard with the same number of light sources, compared with rectangular and circular light source layouts, the layout given in this paper 


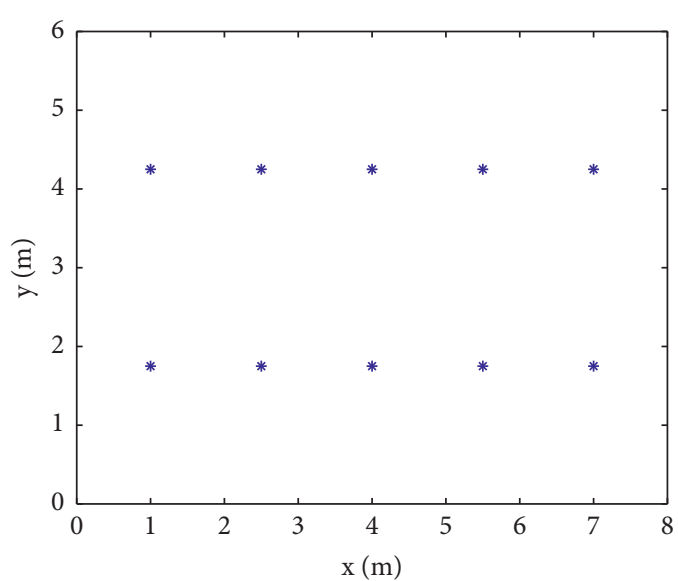

* Light source

(a)

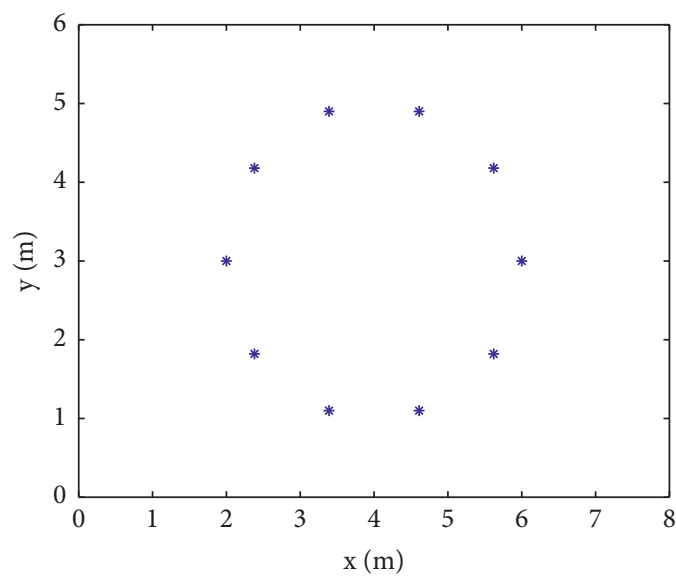

* Light source

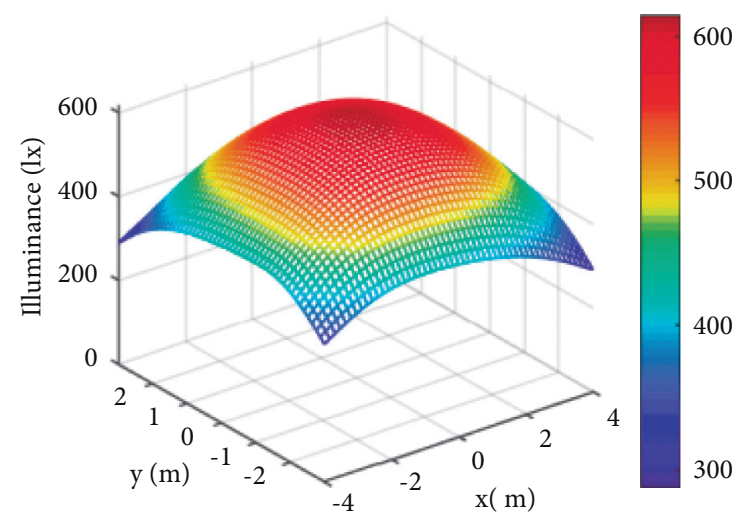

(b)

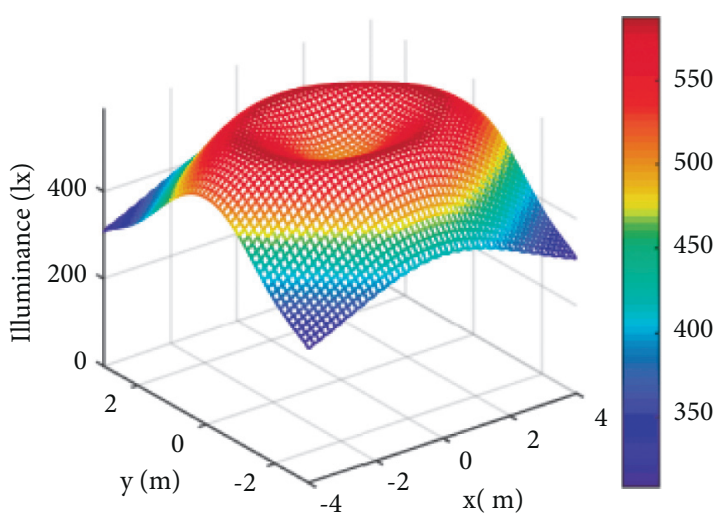

(d)

Figure 11: $8 \mathrm{~m} \times 6 \mathrm{~m} \times 3 \mathrm{~m}$ room layout and illumination diagram. (a) Layout of rectangular light source. (b) Rectangular layout illumination map. (c) Layout of circular light source. (d) Circular layout illumination map.

has the highest illumination uniformity while meeting the lighting standard.

In this section, combined with the illuminance and illuminance uniformity standards, it is proved that the illuminance and illuminance uniformity of the indoor light source layout given by this method is better than the rectangular and circular light source layout given in the current literature.

5.3. Simulation Experiment 3. In order to verify the energy consumption comparison between the light source layout given by this method and the indoor light source layout given by other literatures in the same illumination effect, the rectangular layout, circular layout, and this method can achieve the average illumination effect of $450 \mathrm{~lx}, 550 \mathrm{~lx}$ and $700 \mathrm{~lx}$ in the same room. The number of light sources required for each layout is shown in Tables 4, 5, and 6 .

In the table, in the same room to achieve the same illumination requirements, the number of light sources required for this layout is less than that for rectangular and circular layout. In different rooms to achieve the same illumination, the number of additional light sources required for this layout is less than that for rectangular and circular layout, which proves that this layout can effectively reduce the number of light sources used, which is in line with the environmental protection concept of high efficiency and energy saving.

5.4. Simulation Experiment 4. In order to verify the versatility of the method in this paper, a complex L-shaped room is selected for verification, and its space model and size are shown in Figure 13. Since there is no other relevant literature to design the layout of the light source in a complex room, simulation experiment 4 only analyzes and explains the effect of the layout of the light source in this paper.

The lighting layout design of complex room is different from the traditional rectangular room, which is limited by the conditions such as the superposition of regional light 

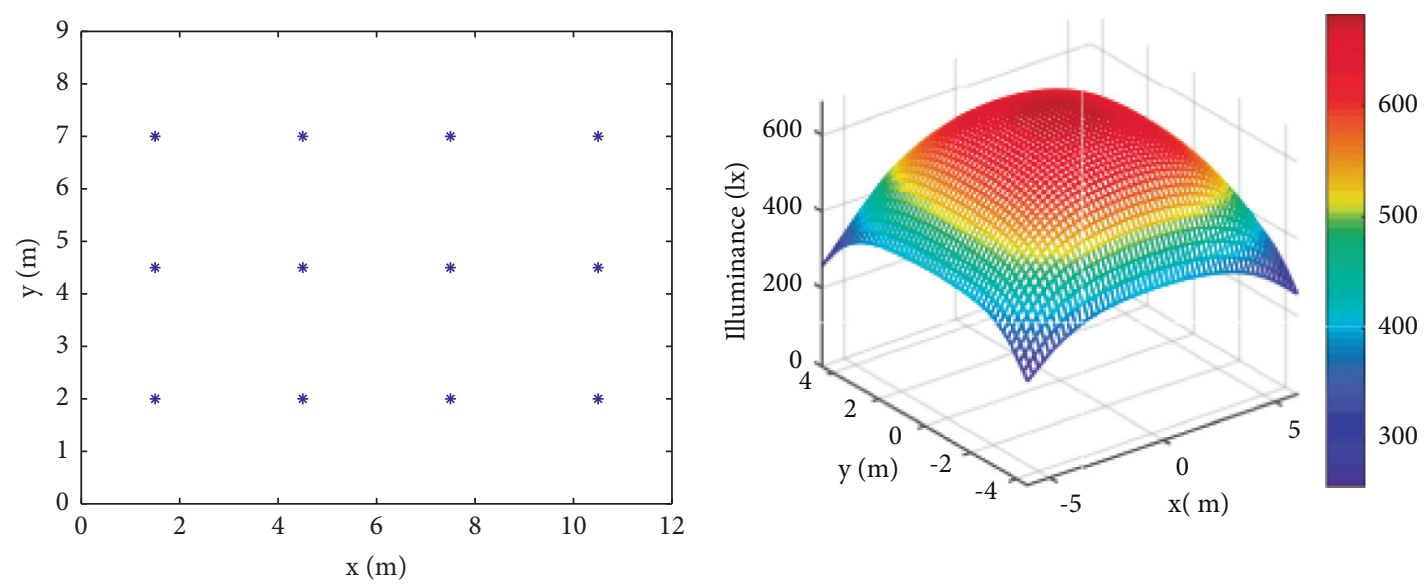

* Light source

(a)

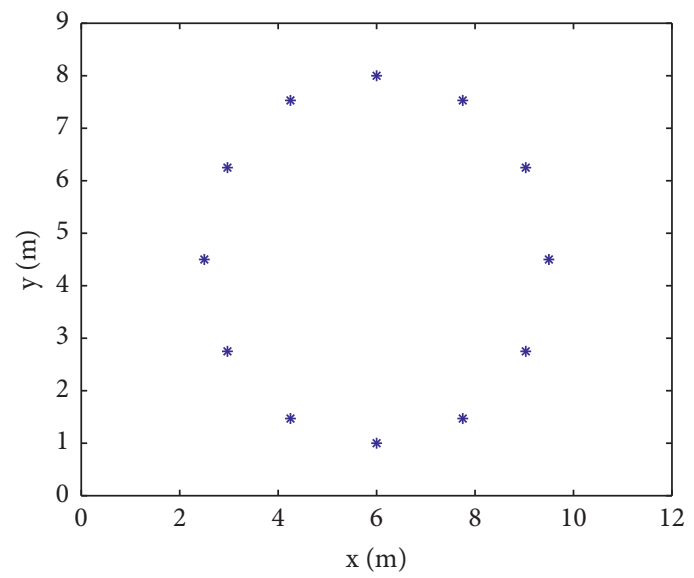

(b)

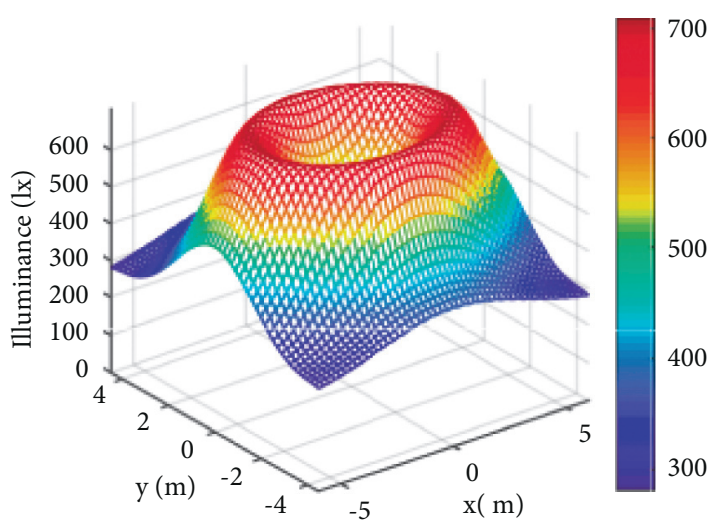

* Light source

(c)

(d)

FIGURE 12: $12 \mathrm{~m} \times 9 \mathrm{~m} \times 3 \mathrm{~m}$ room layout and illumination diagram. (a) Layout of rectangular light source. (b) Rectangular layout illumination map. (c) Layout of circular light source. (d) Circular layout illumination map.

TABLE 3: Illumination uniformity table of different room layout.

\begin{tabular}{lccc}
\hline Room size $(\mathrm{m})$ & Rectangular layout $(\%)$ & Circular layout $(\%)$ & Layout of this paper (\%) \\
\hline $5 \times 5 \times 3$ & 74.2 & 75.7 & 86.4 \\
$8 \times 6 \times 3$ & 72.5 & 71.2 & 82.1 \\
$12 \times 9 \times 3$ & 68.3 & 68.4 & 79.6 \\
\hline
\end{tabular}

TABLE 4: The number of light sources required for each layout in a $5 \times 5 \times 3 \mathrm{~m}$ room.

\begin{tabular}{lccc}
\hline $\begin{array}{l}\text { Illumination value } \\
(\mathrm{lx})\end{array}$ & $\begin{array}{c}\text { Number of light sources in rectangular } \\
\text { layout }\end{array}$ & $\begin{array}{c}\text { Number of light sources in circular } \\
\text { layout }\end{array}$ & $\begin{array}{c}\text { Number of light sources laid out } \\
\text { in } \\
\text { this }\end{array}$ \\
\hline 450 & 9 & 10 & 9 \\
550 & 12 & 12 & 11 \\
700 & 14 & 16 & 13 \\
\hline
\end{tabular}


TABLE 5: The number of light sources required for each layout in a $8 \times 6 \times 3 \mathrm{~m} \mathrm{room}$.

\begin{tabular}{lccc}
\hline $\begin{array}{l}\text { Illumination value } \\
(\mathrm{lx})\end{array}$ & $\begin{array}{c}\text { Number of light sources in rectangular } \\
\text { layout }\end{array}$ & $\begin{array}{c}\text { Number of light sources in circular } \\
\text { layout }\end{array}$ & $\begin{array}{c}\text { Number of light sources laid out } \\
\text { in } \\
\text { this }\end{array}$ \\
\hline 450 & 12 & 13 & 11 \\
550 & 15 & 16 & 14 \\
700 & 16 & 18 & 15 \\
\hline
\end{tabular}

TABLE 6: The number of light sources required for each layout in a $12 \times 9 \times 3 \mathrm{~m}$ room.

\begin{tabular}{|c|c|c|c|}
\hline $\begin{array}{l}\text { Illumination value } \\
(\mathrm{lx})\end{array}$ & $\begin{array}{c}\text { Number of light sources in rectangular } \\
\text { layout }\end{array}$ & $\begin{array}{c}\text { Number of light sources in circular } \\
\text { layout }\end{array}$ & $\begin{array}{l}\text { Number of light sources laid out in } \\
\text { this paper }\end{array}$ \\
\hline 450 & 14 & 15 & 12 \\
\hline 550 & 16 & 17 & 15 \\
\hline 700 & 18 & 20 & 17 \\
\hline
\end{tabular}

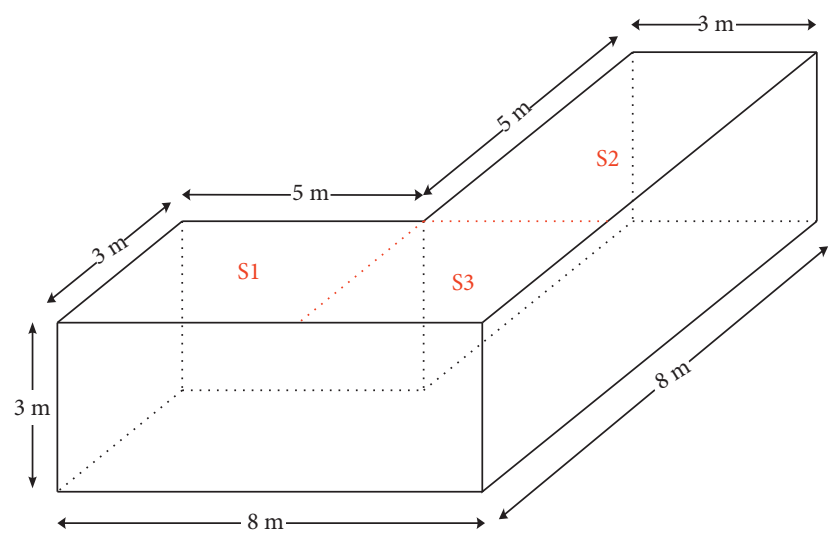

FiguRE 13: Schematic diagram of L-shaped room.

sources and the loss of illumination. For the optimization of light source layout in irregular rooms, the room layout is first cut into regular areas for calculation. As shown in Figure 13, the L-shaped room in this paper is divided into three areas S1, S2, and S3. Since the S3 area light source will affect the S1 and S2 areas, and it will be superimposed by the illuminance of the S1 and S2 area light sources, when calculating the illuminance value of the room, the S1 and S2 areas are prioritized for layout optimization. When calculating the light source superposition region S3, restricted by the illumination of $\mathrm{S} 1$ and $\mathrm{S} 2$ regions, the contour detection and matching of the search region are carried out. The minimum coverage problem [36] is used to solve the problem, and the improved artificial bee colony algorithm is combined to optimize the light source layout of L-shaped complex space. The light source layout and illumination distribution of complex space are shown in Figure 14.

In Figure 14(d), the illuminance distribution of the L-type room is characterized by regional fluctuations due to the division calculation of the space. In S1, S2, and S3 areas, the total coverage of illumination is achieved, and the overlapping area of illumination has a little higher illumination. The maximum illumination is $492.6214 \mathrm{~lx}$, the minimum is $302.3994 \mathrm{~lx}$, and the illumination uniformity is $82.7 \%$. The illumination is within the illumination standard range, the illumination uniformity is much higher than the standard, and the feasibility of the algorithm in indoor complex area is verified.

To sum up, in terms of average illumination and uniformity of illumination, the lighting effect achieved by the layout proposed in this paper meets the lighting standards. Compared with rectangular and circular light source layout [35], the illumination uniformity is higher, the overall illumination difference is lower, and the spatial illumination distribution is more balanced, so less light sources are used to achieve the required illumination. The layout optimized by improved artificial bee colony algorithm not only has more advantages in the effect of indoor lighting, but also can meet the requirements of standard lighting layout for irregular rooms without general light source layout, achieving lighting effect and saving energy. 


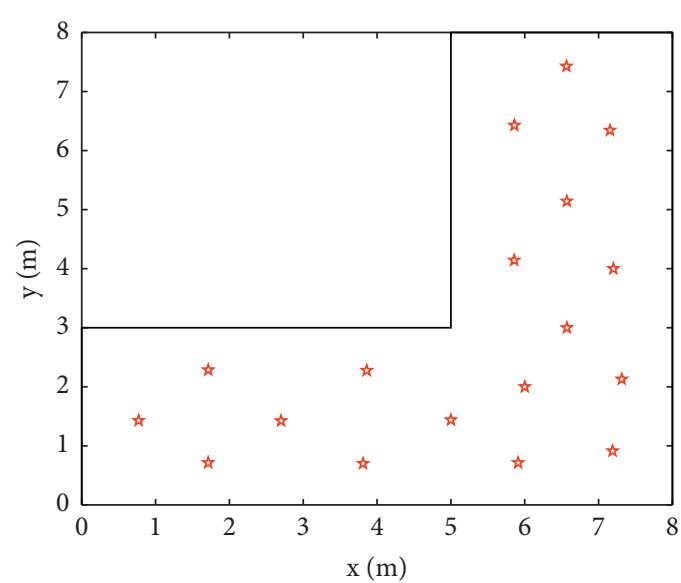

\ Light source

(a)

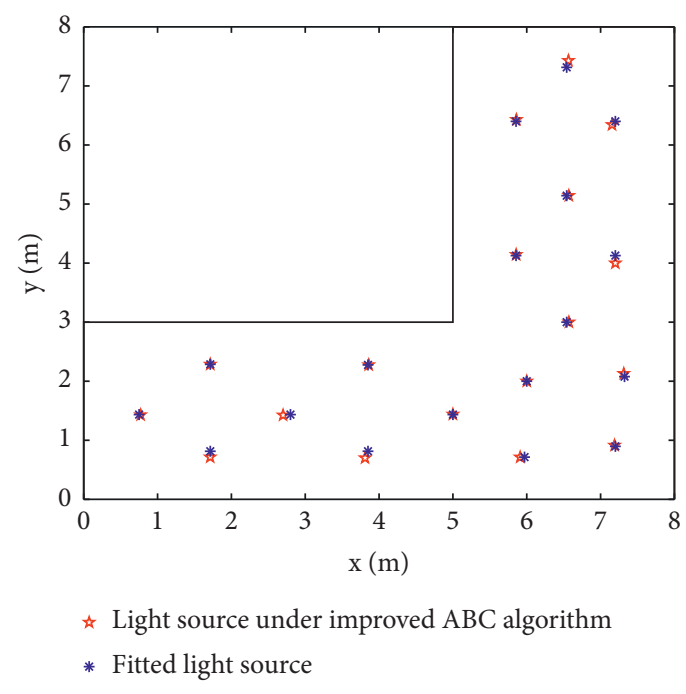

(c)

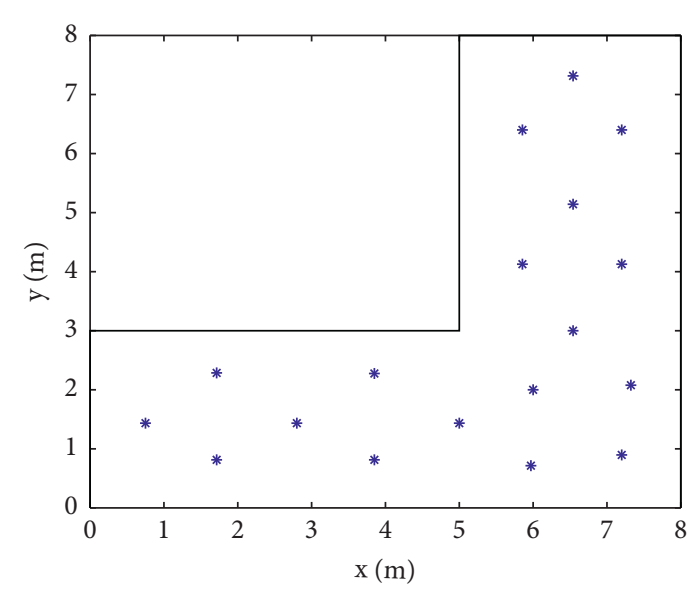

* Light source

(b)

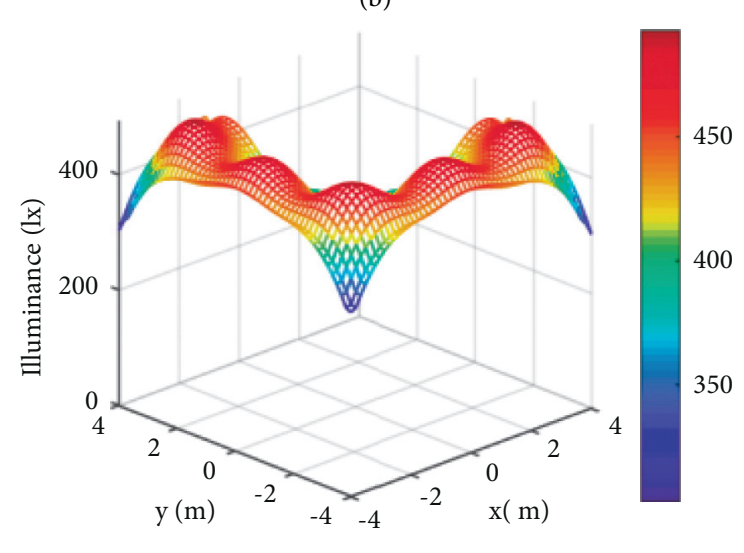

(d)

FIGURE 14: L-type room light source layout and illumination diagram. (a) Optimized layout. (b) Fitted layout. (c) Layout comparison. (d) Ltype room illumination diagram.

\section{Conclusion}

In order to enhance the indoor lighting effect and reduce the energy loss of excess light source, this paper focuses on the layout of indoor light source. The general LED lighting model is selected and analyzed, and its mathematical model is constructed. In order to find the specific lighting position of the light source under the optimal lighting effect, the optimization mechanism of artificial bee colony algorithm is used for reference, and the improved artificial bee colony algorithm combined with region segmentation is proposed to optimize the location and layout of the light source. According to the rationality of light source construction, the specific location of standardized light source is obtained by numerical fitting. Then, in the simulation experiment, the lighting effect of different specifications of the room is compared. In the case of the same illumination and the same number of light sources, it is proved that the advantage of uniform distribution of illumination after the standardized fitting is fully in line with and better than the indoor lighting standard. Through the above experiments, the overall idea of improved artificial bee colony algorithm is proved to be effective, which reduces the energy loss of indoor light source and provides a new choice for indoor light source layout. Although the improved artificial bee colony algorithm has advantages, due to the introduction of the idea of region segmentation algorithm, the algorithm focuses more on region search, which weakens its development ability and consumes too much global search time. How to improve the development ability of the improved artificial bee colony algorithm and reduce its time loss will be the work content in the future. At the same time, based on the experience of using the improved artificial bee colony algorithm to study the light source layout in this paper, in the development of artificial bee colony algorithm, I think the following problems are worth exploring. In the future work, the artificial bee colony algorithm can be combined with more swarm intelligence algorithms. Under the new constrained 
multiobjective optimization problem, to develop a hybrid swarm intelligence algorithm can solve practical problems.

\section{Data Availability}

The data used to support the findings of this study are available from the corresponding author upon request.

\section{Conflicts of Interest}

The authors declare that there are no conflicts of interest.

\section{Acknowledgments}

This work was supported in part by the Natural Science Foundation Program of Liaoning Province of China under Grants J2020109 and 2019-ZD-0289.

\section{References}

[1] L. Carpenter, "Green lights, blue skies," Sewanee Review, vol. 128, no. 2, pp. 189-200, 2020.

[2] D. Vn and K. Va, "Energy saving and LED lamp lighting and human health," Gigiena i Sanitaria, vol. 81, 2013.

[3] A. M. Moram, "Light-emitting diodes and their applications in energy-saving lighting," Proceedings of the Institution of Civil Engineers-Energy, vol. 164, no. 1, 2011.

[4] N. Kumar and N. R. Lourenco, "Led-based visible light communication system: a brief survey and investigation," Journal of Engineering and Applied Sciences, vol. 5, no. 4, pp. 296-307, 2012.

[5] T. Komine and M. Nakagawa, "Fundamental analysis for visible-light communication system using LED lights," IEEE Transactions on Consumer Electronics, vol. 50, no. 1, pp. 100-107, 2004.

[6] S. Hann, J. Kim, S. Jung, and C. Park, "White LED ceiling lights positioning systems for optical wireless indoor applications," in Proceedings of the 36th European Conference and Exhibition on Optical Communication, pp. 1-3, Turin, Italy, September 2010.

[7] T. Komine, S. Haruyama, and M. Nakagawa, "A study of shadowing on indoor visible-light wireless communication utilizing plural white LED lightings," Wireless Personal Communications, vol. 34, no. 1-2, pp. 211-225, 2005.

[8] H. Z. Yang, "Research on the design strategy of children's interior lighting," Light and Lighting, vol. 43, no. 04, pp. 47-50, 2019.

[9] R. J. Wei, L. Lv, and L. C. Yang, "A lighting design algorithm in complex regions," Journal of Computer-Aided Design \& Computer Graphics, vol. 27, no. 10, pp. 1944-1949, 2015.

[10] D. Seo, L. Park, P. Ihm, and M. Krarti, "Optimal electrical circuiting layout and desk location for day lighting controlled spaces," Energy and Buildings, vol. 51, pp. 122-130, 2012.

[11] B. W. Guo and Y. X. Guo, "Optimization design of spatial layout interior environment in point source small disturbance," Bulletin of Science and Technology, vol. 32, no. 01, pp. 128-132, 2016.

[12] A. J. Wang, Y. Che, Y. L. Guo, and L. X. Wang, "LED layout optimization and performance analysis of indoor visible light communication system," Chinese Journal of Lasers, vol. 45, no. 05 , pp. 172-183, 2018.
[13] S. Jin and S. H. Lee, "Lighting layout optimization for 3D indoor scenes," Computer Graphics Forum, vol. 38, no. 7, pp. 733-743, 2019.

[14] H. Wang, H. Q. Zhang, H. H. Wang, and F. X. Zhang, "Design of LED arrays for uniform near-field illumination," Optics \& Optoelectronic Technology, vol. 7, no. 05, pp. 78-83, 2009.

[15] D. Z. Tian, J. S. Li, H. Y. Wang, and D. X. Wang, "Network traffic prediction method based on improved $\mathrm{ABC}$ algorithm optimized EM-ELM," The Journal of China Universities of Posts and Telecommunications, vol. 25, no. 03, pp. 33-44, 2018.

[16] Z. Tian, G. Wang, S. Li, Y. Wang, and X. Wang, "Artificial bee colony algorithm-optimized error minimized extreme learning machine and its application in short-term wind speed prediction," Wind Engineering, vol. 43, no. 3, pp. 263-276, 2019.

[17] Z. Tian, G. Wang, Y. Ren, S. Li, and Y. Wang, "An adaptive online sequential extreme learning machine for short-term wind speed prediction based on improved artificial bee colony algorithm," Neural Network World, vol. 28, no. 3, pp. 191-212, 2018.

[18] C. K. Cowan and A. Bergman, "Determining the camera and light source location for a visual task," in Proceedings of the International Conference on Robotics and Automation, vol. 1, pp. 509-514, Scottsdale, AZ, USA, May 1989.

[19] K. R. Konda and N. Conci, "Illumination modelling and optimization for indoor video surveillance," Computational Imaging XII, vol. 9020, 2013.

[20] C. Cuttle, "Making the switch from task illumination to ambient illumination standards: principles and practicalities, including energy implications," Lighting Research \& Technology, vol. 52, no. 4, pp. 455-471, 2020.

[21] J. Vitasek, T. Stratil, J. Latal, J. Kolar, and Z. Wilcek, "Indoor illumination imitating optical parameters of sunny summer daylight," Optics and Laser Technology, vol. 124, 2020.

[22] M. B. Kosfic and F. V. Topalis, "Interior lighting calculations: survey of theoretical methods," Lighting Research and Technology, vol. 30, no. 4, pp. 151-157, 1998.

[23] D. Karaboga and B. Basturk, "On the performance of artificial bee colony (ABC) algorithm," Applied Soft Computing Journal, vol. 8, no. 1, pp. 687-697, 2007.

[24] B. Akay and D. Karaboga, "A modified artificial bee colony algorithm for real-parameter optimization," Information Sciences, vol. 192, pp. 120-142, 2012.

[25] B. Zhang, T. T. Liu, S. C. Zhang, and P. Wang, “Artificial bee colony algorithm with strategy and parameter adaptation for global optimization," Neural Computing and Applications, vol. 28, no. 1, pp. 349-364, 2017.

[26] W. Ma, Z. Sun, J. Li, M. Song, and X. Lang, "An improved artificial bee colony algorithm based on the strategy of global reconnaissance," Soft Computing, vol. 20, no. 12, pp. 4825-4857, 2016.

[27] S. Babaeizadeh and R. Ahmad, "Performance comparison of constrained artificial bee colony algorithm," Research Journal of Applied Sciences, Engineering and Technology, vol. 10, no. 5, pp. 537-546, 2015.

[28] A. Yurtkuran and E. Emel, "An enhanced artificial bee colony algorithm with solution acceptance rule and probabilistic multisearch," Computational Intelligence and Neuroscience, vol. 2016, Article ID 8085953, 13 pages, 2016.

[29] J. Liu, H. Zhu, Q. Ma, L. Zhang, and H. Xu, “An artificial bee colony algorithm with guide of global and local optima and 
asynchronous scaling factors for numerical optimization," Applied Soft Computing, vol. 37, pp. 608-618, 2015.

[30] J. X. Bi and J. R. Gong, "Hybrid clustering algorithm based on artificial bee colony and K-means algorithm," Application Research of Computers, vol. 29, no. 6, pp. 2040-2042, 2012.

[31] L. L. Long, T. P. Lee, and S. Y. Whar, "Direct least squares fitting of ellipses segmentation and prioritized rules classification for curve-shaped chart patterns," Applied Soft Computing Journal, vol. 107, 2021.

[32] M. Žic, "An alternative approach to solve complex nonlinear least-squares problems," Journal of Electroanalytical Chemistry, vol. 760, pp. 85-96, 2016.

[33] A. Amiri-Simkooei and S. Jazaeri, "Weighted total least squares formulated by standard least squares theory," Journal of Geodetic Science, vol. 2, no. 2, pp. 113-124, 2012.

[34] W. Wanguo, W. Shirong, X. Zhengfei, Y. Wenbo, W. Zhenli, and L. Li, "Improved least squares ellipse fitting algorithm based on boundary," Computer Technology and Development, vol. 23, no. 4, pp. 67-70, 2013.

[35] T. H. Do, J. Hwang, and M. Yoo, "Analysis of the effects of LED direction on the performance of visible light communication system," Photonic Network Communications, vol. 25, no. 1, 2013.

[36] H. M. Li, Algorithm Research on Minimum Power Partial Cover Problem, Zhejiang Normal University, Jinhua, China, 2020. 